www.jmscr.igmpublication.org

Index Copernicus Value: 79.54

ISSN (e)-2347-176x ISSN (p) 2455-0450

crossref DOI: https://dx.doi.org/10.18535/jmscr/v7i6.89

\title{
Gender difference and therapeutic effectiveness of platelets rich plasma intra-articular injections among elderly with knee osteoarthritis
}

\author{
Authors
}

\section{Hala Samir Sweed ${ }^{1}$, Maram Maher Monier ${ }^{2}$, Mohamed Ragaai El -Helow ${ }^{3}$,} Khaled Mohamed Emara ${ }^{4}$, Mohammad A. Zakaria ${ }^{5}$, Rana Mohammed Taha ${ }^{6}$

${ }^{1,2,6}$ Department of Geriatrics and Gerontology, Faculty of Medicine - Ain Shams University. Cairo, Egypt

${ }^{3,5}$ Department of Physical Medicine, Rheumatology and Rehabilitation, Faculty of Medicine - Ain Shams

University, Cairo, Egypt

${ }^{4}$ Department of Orthopedic Surgery, Faculty of Medicine - Ain Shams University, Cairo, Egypt

*Corresponding Author

Maram Maher Monier

Department of Geriatrics and Gerontology, Faculty of Medicine - Ain Shams University, Cairo, Egypt

\begin{abstract}
Background: Osteoarthritis $(O A)$ is the most prevalent etiology for arthritis. Knee OA clinical symptoms and signs have direct impact on social interactions, mental functioning, sleep quality and various aspects of patients' lives. The applications of biologic managing agents such as PRP in musculoskeletal disorders are growing considerably.

Aim: To investigate and compare gender differences in response to platelets rich plasma intra articular injections in cases having knee osteoarthritis.

Methodology: The research clinical trial recruited 44 elderly participants aged sixty years and above with mild to moderate knee osteoarthritis according to Kellgren-Lawrence Grading Scale system. The studied sample have been categorized into two equal matched age research groups: Research Group A:22 male patients 60 years old and above with knee osteoarthritis. Research Group B:22 female patients 60 years old and above with knee osteoarthritis.

Results: Significant improvement in pain scores among all grades of knee OA, the best improvement achieved at 6 months follow up. Rate of improvement in pain Scores at 6 and 12 months follow up was almost equal between the 3 grades of $O A$ with no statistically significant difference.

Conclusions and Recommendations: Intra-articular injection of PRP in knee OA may be a valid alternative treatment for knee osteoarthritis. Intra-articular infiltration of autologous PRP under the conditions described in this study seems to be a safe, effective treatment for knee OA and does not require hospitalization or surgery.

Keywords: platelets rich plasma, knee osteoarthritis, elderly.
\end{abstract}

\section{Introduction}

Osteoarthritis (OA) is the most common cause of arthritis, Knee $\mathrm{OA}$ is the chief clinical presentation of $\mathrm{OA}$, being the leading cause of musculoskeletal disability all over the globe among the elderly age groups. ${ }^{1,2}$

Knee OA clinical symptoms and signs have direct impact on social interactions, mental functioning, 
sleep quality and various aspects of patients' lives. Restrictions in walking, stair climbing and squatting are frequent complaints interfering and restricting greatly cases daily activities in a manner affecting the life style Osteoarthritis pathophysiological development is gradually progressive overtime on the other hand staying active, maintaining a healthy weight in conjunctions with other management protocols could slow pathological disease progression aiding in improvement of pain and joint function issues. $^{3,4}$

Various agents are implemented to relief the with knee osteoarthritis cases symptoms e.g. NSAIDS, glucosamine, and chondroitin-sulfate, intraarticular injections (glucocorticoids, hyaluronic acid), biophysical supporting and managing tools (e.g. appropriate braces, shoes and insoles, exercise therapy, laser therapy, application of heat and cold modalities, etc.), and corrective surgical interventions. It seems that existing treatments cannot change the pathophysiological disease process. $^{5,6}$

Modern therapeutically methods trigger cartilaginous healing process and such as matrix metalloproteinase inhibitors, gene therapy, cytokinase inhibitors, stem cells, and growth factors. The platelet growth factors trigger the healing process causing partial modification of the damaged tissue. Platelet-rich plasma (PRP), with higher platelet concentration levels than the mean blood measures, is considered one of the sources for growth factors. ${ }^{7,8}$

These usage of growth factors within platelet-rich plasma (PRP), obtained from centrifugation of autologous blood and applied to the site of tissue lesions to trigger and accelerate physiological healing processes. The tissues of orthopedic interest that undergo cellular regeneration are cartilage, tendons, ligaments, muscle, and bone. $^{9,10}$

PRP is a simple, economic, and minimally invasive therapy that provides a concentrate of autologous growth factors which can be used to activate and accelerate the physiological processes of healing. Activated platelets release growth factors contained in their granules. In this way, the plasma becomes a vehicle of growth factors which play key roles in the process of healing of many tissues. $^{11,12}$

The applications of biologic managing agents such as PRP in musculoskeletal disorders are growing considerably. Although comparing PRP with other intra-articular and soft tissue injections has led to conflicting research results and debate requiring research efforts to elucidate useful effects of PRP on healing and functional improvement of injured tissues. ${ }^{13,14}$

\section{Aim of the work}

To investigate and compare gender differences in response to platelets rich plasma intra articular injections in cases having knee osteoarthritis.

\section{Methodology}

One arm clinical trial was conducted to study the efficacy of PRP injections in elderly and to assess gender difference response to PRP intra articular injections in elderly patients with knee osteoarthritis. In this study about 250 patients attended outpatient clinics and inpatients wards of Ain Shams University Hospital were examined and the diagnosis of bilateral knee osteoarthritis disease was established. Among those patients a sample of 44 research study subjects (22 females and 22 males) fulfilling inclusion and exclusion research study criteria were randomly selected by simple random sampling.

The study sample comprised 44 elderly participants aged sixty years and above with mild to moderate knee osteoarthritis according to Kellgren-Lawrence Grading Scale system The studied sample have been categorized into two equal matched age research groups: Research Group A: 22 male patients 60 years old and above with knee osteoarthritis. Research Group B: 22 female patients 60 years old and above with knee osteoarthritis.

Sample size have been calculated assuming a change of WOMAC score ranging between 26 and 
$30+/-8 \mathrm{SD}$, a sample size of 44 is enough to detect such difference based on 0.05 alpha over 0.090 power of the test.

Exclusive research criteria:Participants who refuse to participate in the study, Body mass index $\geq 35$,severe knee osteoarthritis (grade 4; large osteophytes, marked joint space narrowing, severe sclerosis and definite bony deformity as defined by Kellgren and Lawrence system for classification of knee osteoarthritis, active knee injury or acute inflammation or infection ,bleeding disorders (platelets disorders, coagulopathies). Cases on (antiplatelet / anticoagulation) therapy at least for 10 days before injection .Medical history of immunodeficiency disorders, malignancies, autoimmune diseases or uncontrolled diabetes mellitus, past history of knee articular injections of corticosteroids during previous month or use of systemic corticosteroid 2 weeks before PRP injections, cases depressed or cognitively impaired since it impairs proper assessment of pain and physical function.

For each study subject the following was performed Informed consent taking taken from the participant after explanation of the study aim and procedures. Comprehensive Geriatric Assessment involving full medical history, full Clinical examination involving detailed knee joint examination; inspecting the affected knee for erythema, swelling, bruising, discoloration, and musculature. then palpated and checked for pain, warmth, and effusion and point tenderness, range of motion was assessed by extending and flexing the knee as far as possible. Activity of the daily living (ADL) performed at $0 \mathrm{~ms}, 6 \mathrm{~ms}$ and $12 \mathrm{~ms}$ post injection was used to rank adequacy of performance in six functions: bathing, dressing, toileting, transferring, continence, and feeding. If no supervision, direction, or personal assistance is required, then 1 point is given to that functional activity. If patient requires supervision, direction, personal assistance, or total care, then a 0 is assigned to that functional activity. A total score of 6 indicate High (patient is independent) and score of 0 indicate Low (patient is totally dependent).

Western Ontario and McMaster Universities Arthritis Index (WOMAC) questionnaire. It was done $0 \mathrm{~ms}$, at $6 \mathrm{~ms}$ and $12 \mathrm{~ms}$ post injection: To assess pain, stiffness, and physical function in patients with knee osteoarthritis.

Laboratory investigations: $\mathrm{CBC}$ and Bleeding Profile. (PTT, PT, INR) were done before injection at central laboratories of Ain Shams university hospital to exclude platelets disorders and bleeding tendency.

Radiographic assessment: Plain $\mathrm{X}$ ray AP \& lateral weight bearing was done at baseline only to assess the grade of OA in the patients according to Kellgren-Lawrence Grading scaling system.

The study procedure (preparation, injection and follow up): preinjection preparation of platelets rich plasma (PRP): For each injection, venesection of $30 \mathrm{ml}$ venous blood from the medial cubital vein was done using a butterfly cannula (19-21 gauges) connected to a $50 \mathrm{ml}$ syringe with gentle suction. The blood is drawn into a sterilized $50 \mathrm{ml}$ falcon tube containing $5 \mathrm{ml}$ of anticoagulation citrate dextrose-A solution (ACD-A). The aspirated blood was gently shaken up to thoroughly mix the anticoagulant with the blood. Using the centrifuge device (Centerion 2006®, England), two centrifugations (the first at $1800 \mathrm{rpm}$ for $15 \mathrm{~min}$ to separate erythrocytes, and a second at $3500 \mathrm{rpm}$ for $10 \mathrm{~min}$ to concentrate platelets) produced a unit of $5 \mathrm{ml}$ of PRP. A puffy coat (which is the layer between the stagnant red layer of RBCs and the straw-colored layer of plasma) is aspirated using a $10 \mathrm{ml}$ syringe. All the procedures were performed in the same office setting in a sterile hood. Prior the injection, $0.5 \mathrm{ml}$ of $10 \%$ of Ca-chloride was added to the PRP unit $(1: 10)$ to activate platelets.

Injection of PRP: 2-3 $\mathrm{ml}$ of PRP was injected back into each affected knee joint in a single session either by classic lateral or ventral approaches of intra-articular injection under complete aseptic conditions using specialized injection needle. The patient is supine on the 
table, with a rolled towel under the knee to relax the knee muscles. The sterile needle inserted lateral or medial to the patellar tendon approximately $1 \mathrm{~cm}$ above the tibial plateau and directed $15-45^{\circ}$ from anterior knee surface vertical midline toward the intraarticular joint space. Immediately after the injection, passive flexion and extension of the injected knee was performed three times, followed by 10 minutes of resting supine patients were instructed to exert only mild effort by the injected legs for 24 hours and to use ice packs over the injected joint. During the period of treatment, the patient is forbidden to take nonsteroidal anti-inflammatory drugs (NSAIDs). In the case of pain onset, they had permission to use $500 \mathrm{mg}$ of acetaminophen only. Also, they were instructed not to adhere to any physical therapy programs.

Post injection follow up: There was no added specific treatment during the 12 months period of follow up. Patients were re interviewed at $6 \mathrm{~ms}$ and $12 \mathrm{~ms}$ of follow up period. First and Second stages of follow up has been done by different interviewers who were blinded to results of each other to avoid results bias. Then results were compared to the base line scores to assess the effect of PRP injections.

In the study patients were subjected to assessment at baseline, 6 months, and 12 months post injection by:

1. Body mass index (BMI) measuring.

2. Activity of the daily living (ADL).

3. Timed up and Go test.

4. Numeric pain rating scale (NRS-11).

5. Western Ontario and McMaster Universities Arthritis Index (WOMAC) questionnaire.

Ethical consideration: The research was carried out after the approval of The Research Ethics Committee; Faculty of Medicine, Ain Shams University. (REC- FMASU) monitoring board.

\section{Statistical Analysis}

Data were collected, revised, coded, and entered to the Statistical Package for Social Science (IBM SPSS) version 23. Qualitative variables were presented as number and percentages and comparison between groups regarding qualitative data was done by using Chi-square test and/or Fisher exact test when the expected count in any cell found less than 5 . The confidence interval was set to $95 \%$ and the margin of error accepted was set to $5 \%$. So, the p-value was considered significant at the level of $<0.05$.

\section{Results}

Table (1): Demographic data and clinical characteristics of the study sample

Table (1a):

\begin{tabular}{|c|c|}
\hline Variable & $\begin{array}{c}\text { Count } \\
\text { (Percentage) }\end{array}$ \\
\hline $\begin{array}{l}\text { Gender } \\
\\
\text { Males: } \\
\text { Females: }\end{array}$ & $\begin{array}{l}22(50 \%) \\
22(50 \%)\end{array}$ \\
\hline $\begin{array}{l}\text { Marital status } \\
\text { Married: } \\
\text { Divorced: } \\
\text { Widowed: }\end{array}$ & $\begin{array}{c}34(77.27 \%) \\
4(9.09 \%) \\
6(13.64 \%)\end{array}$ \\
\hline $\begin{array}{l}\text { Not working: } \\
\text { Employed: } \\
\text { Retired: }\end{array}$ & $\begin{array}{c}20(45.45 \%) \\
6(13.64 \%) \\
18(40.91 \%) \\
\end{array}$ \\
\hline $\begin{array}{l}\text { Education } \\
\text { Illiterate: } \\
\text { Below high school: } \\
\text { High school: } \\
\text { Higher education: } \\
\end{array}$ & $\begin{array}{c}20(45.45 \%) \\
8(18.18 \%) \\
5(11.36 \%) \\
11(25.00 \%) \\
\end{array}$ \\
\hline $\begin{array}{l}\text { Smoking } \\
\qquad \begin{array}{l}\text { Smoker: } \\
\text { Non smoker: }\end{array}\end{array}$ & $\begin{array}{l}15(34.09 \%) \\
29(65.91 \%)\end{array}$ \\
\hline $\begin{array}{c}\text { OA grade: } \\
\text { Grade I } \\
\text { Grade II } \\
\text { Grade III }\end{array}$ & $\begin{array}{l}8(18.18 \%) \\
17(38.64 \%) \\
19(43.18 \%)\end{array}$ \\
\hline $\begin{array}{c}\text { Chronic diseases } \\
\text { DM } \\
\text { HTN } \\
\text { ISHD } \\
\text { COPD } \\
\text { BA } \\
\text { Osteoporosis } \\
\text { CLD } \\
\end{array}$ & $\begin{array}{c}14(31.82 \%) \\
17(38.64 \%) \\
10(22.73 \%) \\
3(6.82 \%) \\
1(2.27 \%) \\
1(2.27 \%) \\
2(4.55 \%)\end{array}$ \\
\hline
\end{tabular}


Table (1b)

\begin{tabular}{|c|ccc|rcc|}
\hline \multicolumn{7}{|c|}{ Descriptive Statistics } \\
\hline \hline & \multicolumn{9}{|c|}{ Range } & Mean & \pm & SD \\
\hline Age & 60 & - & 70 & 64.091 & \pm & 4.074 \\
\hline Height & 155 & - & 182 & 167.841 & \pm & 6.765 \\
\hline Weight & 65 & - & 100 & 82.500 & \pm & 7.795 \\
\hline BMI & 24.2 & - & 33.3 & 29.343 & \pm & 2.628 \\
\hline
\end{tabular}

Table (1) shows that:

We have 2 equal groups of males and females each include 22 subjects, $77.27 \%$ are married, $65.91 \%$ illiterate, $45.45 \%$ are not working and nonsmoker. $43.18 \%$ have grade 3 knee OA (table 1a). The participant of the current study age ranged from $60-70$ years with the mean age is 64 years. BMI was measured and its mean value is
29.3 (table 1b). The mean score of ADL is at baseline (5.4), after 6 and 12 months follow up are (5.7) and (5.4) respectively. The mean TUG score at baseline evaluation is (7.6) seconds, (13.5) seconds after 6 months and (15.7) seconds after 12 months.NRS mean scores are (8) at baseline evaluation, (3) after 6 months and (6.9) after 12 months.

Table (2): Comparison of ADL, TUG and NRS pre \& post injection among the studied cases

\begin{tabular}{|c|c|c|c|c|c|}
\hline & \multirow{2}{*}{ Time } & \multicolumn{2}{|c|}{ Paired Differences } & \multicolumn{2}{|c|}{ Paired Samples Test } \\
\hline & & Mean & SD & $\mathbf{T}$ & P-value \\
\hline \multirow{3}{*}{ ADL } & $\mathrm{B} / 6 \mathrm{M}$ & -0.318 & 0.601 & -3.510 & $0.001 *$ \\
\hline & $\mathrm{B} / \mathbf{1 2 M}$ & -0.023 & 0.151 & -1.000 & 0.323 \\
\hline & $6 \mathrm{M} / 12 \mathrm{M}$ & 0.295 & 0.594 & 3.301 & $0.002 *$ \\
\hline \multirow{3}{*}{ TUG } & $\mathrm{B} / 6 \mathrm{M}$ & 4.068 & 1.576 & 17.123 & $<0.001 *$ \\
\hline & $\mathrm{B} / \mathbf{1 2 M}$ & 1.932 & 1.453 & 8.819 & $<0.001 *$ \\
\hline & $6 \mathrm{M} / 12 \mathrm{M}$ & -2.136 & 1.231 & -11.510 & $<0.001 *$ \\
\hline \multirow{3}{*}{ NRS } & $\mathrm{B} / 6 \mathrm{M}$ & 4.795 & 0.878 & 36.223 & $<0.001 *$ \\
\hline & $\mathrm{B} / \mathbf{1 2 M}$ & 1.114 & 0.813 & 9.085 & $<0.001 *$ \\
\hline & 6M/12M & -3.682 & 0.883 & -27.651 & $\bar{c}^{<0.001 *}$ \\
\hline
\end{tabular}

ADL: activities of daily living test, TUG: time up and go test, NRS: numeric rating pain scale, B: before injection

Table (2) shows the comparison between outcome in ADL, TUG and NRS scores at baseline, 6 months, and 12 months post PRP injection among studied population:

ADL scores show significant improvement at 6 months follow up after PRP injection with statistically significant difference $(\mathrm{P}=0.001)$. Also, ADL scores show improvement at 12 months follow up but with no significant statistical difference $(\mathrm{P}=>0.05)$.TUG and NRS scores at 6 months and 12 months follow up show significant improvement with high statistically significant difference $(\mathrm{P}=<0.001)$ over baseline score. In addition, the significant improvement in TUG and NRS scores are better after 6 months than 12 months with high statistically significant difference $(\mathrm{P}=<0.001)$. 
Table (3): Western Ontario and McMaster Universities Arthritis Index (WOMAC) and subscales scores pre and post PRP injection:

\begin{tabular}{|c|c|c|c|c|c|}
\hline & \multirow{2}{*}{ Time } & \multicolumn{2}{|c|}{ Paired Differences } & \multicolumn{2}{|c|}{ Paired Samples Test } \\
\hline & & Mean & SD & $\mathbf{T}$ & P-value \\
\hline \multirow{3}{*}{$\begin{array}{l}\text { WOMAC } \\
\text { P }\end{array}$} & $\mathrm{B} / 6 \mathrm{M}$ & 5.489 & 1.430 & 35.997 & $<0.001 *$ \\
\hline & $\mathrm{B} / \mathbf{1 2 M}$ & 1.818 & 1.466 & 11.631 & $<0.001 *$ \\
\hline & 6M/12M & -3.670 & 1.624 & -21.202 & $<0.001 *$ \\
\hline \multirow{3}{*}{$\begin{array}{l}\text { WOMAC } \\
\text { S }\end{array}$} & $\mathrm{B} / 6 \mathrm{M}$ & 2.364 & 0.833 & 26.619 & $<0.001 *$ \\
\hline & $\mathrm{B} / \mathbf{1 2 M}$ & 0.898 & 0.695 & 12.109 & $<0.001 *$ \\
\hline & 6M/12M & -1.466 & 0.787 & -17.471 & $<0.001 *$ \\
\hline \multirow{3}{*}{$\begin{array}{l}\text { WOMAC } \\
\text { PF }\end{array}$} & $\mathrm{B} / 6 \mathrm{M}$ & 17.727 & 5.807 & 28.639 & $<0.001 *$ \\
\hline & $\mathrm{B} / \mathbf{1 2 M}$ & 5.625 & 3.785 & 13.940 & $<0.001 *$ \\
\hline & 6M/12M & -12.102 & 4.431 & -25.622 & $<0.001 *$ \\
\hline \multirow{3}{*}{$\begin{array}{l}\text { WOMAC } \\
\text { Total }\end{array}$} & B/6M & 25.045 & 7.482 & 31.403 & < $<0.001 *$ \\
\hline & $\mathrm{B} / \mathbf{1 2 M}$ & 7.455 & 6.556 & 10.666 & $<0.001 *$ \\
\hline & 6M/12M & -17.591 & 5.682 & -29.040 & $<0.001 *$ \\
\hline
\end{tabular}

WOMAC: Western Ontario and McMaster Universities Arthritis Index, P:pain, S: stiffness, PF: physical function, B: before.

Table (3) shows the comparison of WOMAC scores for pain, stiffness and physical function subscales before, after 6 and after 12 months of PRP injection, significant improvement in all parameters at 6 month and 12 month of follow up occurred with high statistically significant difference $(\mathrm{P}=<0.001)$. This improvement is significantly better at 6 month follow up than 12 months

Table (4): Comparison among females at baseline, after 6 month and 12-month post injection

\begin{tabular}{|c|c|c|c|c|c|}
\hline & Female & & $\begin{array}{l}\text { ne } \\
\text { S }\end{array}$ & & Paired Test \\
\hline \multirow{3}{*}{ Weight } & B/6M & 0.250 & \pm & 0.629 & 0.076 \\
\hline & $\mathrm{B} / \mathbf{1 2 M}$ & 0.545 & \pm & 0.912 & $0.011 *$ \\
\hline & $6 \mathrm{M} / 12 \mathrm{M}$ & 0.295 & \pm & 0.766 & 0.085 \\
\hline \multirow{3}{*}{ BMI } & $\mathrm{B} / 6 \mathrm{M}$ & 0.082 & \pm & 0.187 & 0.053 \\
\hline & $\mathrm{B} / 12 \mathrm{M}$ & 0.173 & \pm & 0.364 & $0.037 *$ \\
\hline & $6 \mathrm{M} / 12 \mathrm{M}$ & 0.091 & \pm & 0.312 & 0.186 \\
\hline \multirow{3}{*}{ ADL } & $\mathrm{B} / 6 \mathrm{M}$ & -0.318 & \pm & 0.568 & $0.016 *$ \\
\hline & $\mathrm{B} / 12 \mathrm{M}$ & -0.045 & \pm & 0.213 & 0.329 \\
\hline & $6 \mathrm{M} / 12 \mathrm{M}$ & 0.273 & \pm & 0.550 & $0.030 *$ \\
\hline \multirow{3}{*}{ TUG } & $\mathrm{B} / 6 \mathrm{M}$ & 4.045 & \pm & 1.327 & $<0.001 *$ \\
\hline & $\mathrm{B} / \mathbf{1 2 M}$ & 1.455 & \pm & 1.405 & $<0.001 *$ \\
\hline & $6 \mathrm{M} / 12 \mathrm{M}$ & -2.591 & \pm & 1.008 & $<0.001 *$ \\
\hline \multirow{3}{*}{ NRS } & $\mathrm{B} / \mathbf{6 M}$ & 4.636 & \pm & 0.953 & $<0.001 *$ \\
\hline & $\mathrm{B} / \mathbf{1 2 M}$ & 0.955 & \pm & 0.722 & $<0.001 *$ \\
\hline & $6 M / 12 M$ & -3.682 & \pm & 0.945 & $<0.001^{*}$ \\
\hline
\end{tabular}

BMI: body mass index, ADL: activities of daily living, TUG: timed up and go test, NRS: numeric pain rating scale, B: before

Table (4) shows that among studied female population:

ADL scores significantly improved at 6 month and 12-month post injection with statistical significance difference $(\mathrm{P}=<0.05)$ and better improvement achieved at the 6 months follow up.TUG and NRS scores show high statistically significant improvement $(\mathrm{P}=<0.001)$ at 6 month and 12 months follow up with better improvement achieved at 6 months. Loss of weight and lower BMI among females occurred at 12 month follow up which have a statistically significant difference. 
Table (5): Comparison among males at baseline, after 6 month and 12-month post injection

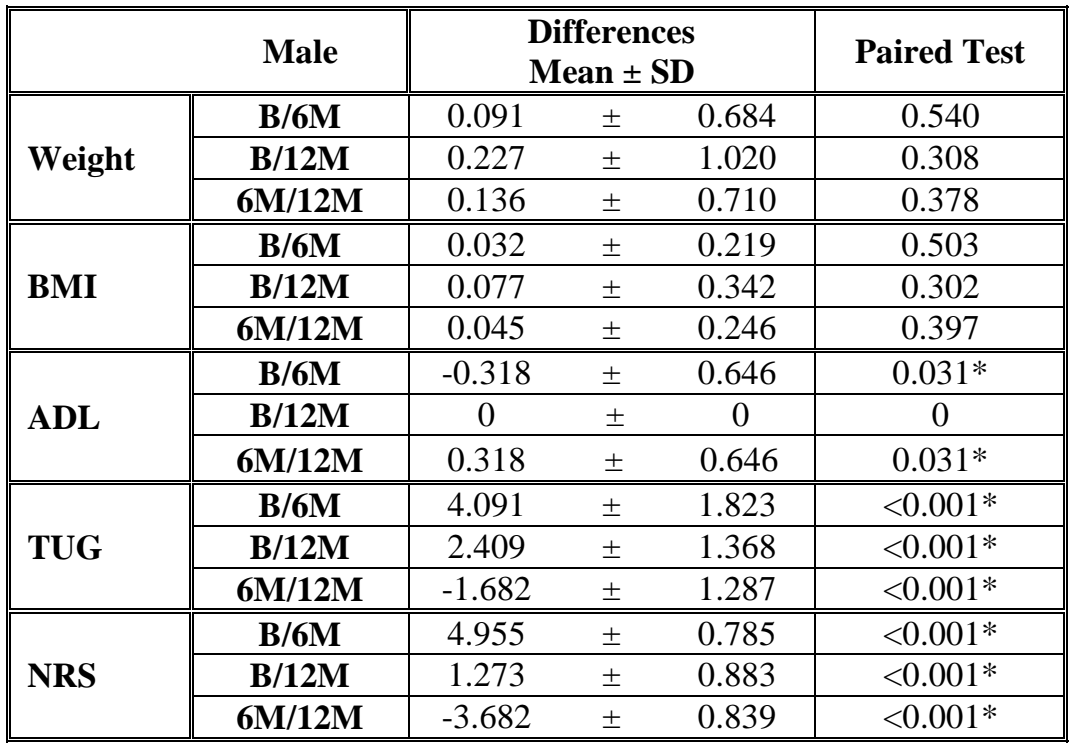

BMI: body mass index, ADL: activities of daily living, TUG: timed up and go test, NRS: numeric pain rating scale, B: before

Table (5) shows that among male Patients. ADL scores significantly improved at 6 month and 12month post injection with statistical significance difference $(\mathrm{P}=<0.05)$ and better improvement achieved at the 6 months follow up.TUG and NRS scores show high statistically significant improvement $(\mathrm{P}=<0.001)$ at 6 month and 12 months follow up with better improvement achieved at 6 months. Unlike females group BMI shows no significant difference.

Table (6): Comparison between males and females as regard ADL, TUG, and NRS pre and post injection

\begin{tabular}{|c|c|c|c|c|c|c|c|c|c|}
\hline & & \multirow{2}{*}{\multicolumn{6}{|c|}{ Sex }} & & \\
\hline & & & & & & & & \multirow{2}{*}{\multicolumn{2}{|c|}{ T-Test }} \\
\hline & & \multicolumn{3}{|c|}{ Female } & \multicolumn{3}{|c|}{ Male } & & \\
\hline & & Mean & \pm & SD & Mean & \pm & SD & $\bar{T} \mathbf{T}$ & P-value \\
\hline \multirow{3}{*}{ ADL } & Before & 5.318 & \pm & 1.041 & 5.545 & \pm & 0.963 & -0.752 & 0.456 \\
\hline & After 6Months & 5.636 & \pm & 0.581 & 5.864 & \pm & 0.468 & -1.429 & 0.160 \\
\hline & After 12Months & 5.364 & \pm & 0.953 & 5.545 & \pm & 0.963 & -0.629 & 0.532 \\
\hline \multirow{3}{*}{ TUG } & Before & 18.682 & \pm & 4.075 & 16.636 & \pm & 5.728 & 1.365 & 0.180 \\
\hline & After 6Months & 14.636 & \pm & 3.360 & 12.545 & \pm & 4.317 & 1.793 & 0.080 \\
\hline & After 12Months & 17.227 & \pm & 3.866 & 14.227 & \pm & 4.947 & 2.241 & $0.030 *$ \\
\hline \multirow{3}{*}{ NRS } & Before & 8.318 & \pm & 1.211 & 7.818 & \pm & 1.435 & 1.249 & 0.219 \\
\hline & After 6Months & 3.682 & \pm & 1.171 & 2.864 & \pm & 1.082 & 2.407 & $0.021 *$ \\
\hline & After 12Months & 7.364 & \pm & 1.432 & 6.545 & \pm & 1.683 & 1.737 & 0.090 \\
\hline
\end{tabular}

ADL: activities of daily living, TUG: time up and go test, NRS: numeric pain rating scale

Table (6) shows that:

TUG scores improved at 6 month and 12 months follow up in both males and females, but the improvement was better at 6 months than 12month post injection among males more than female with no statistically significant difference. TUG scores improvement in male subjects at 12 month of follow up is better than female subjects with high statistical significance difference $(\mathrm{P}=<$
0.05).Improvement in NRS scores also occurred in both males and females at follow up period, improvement is better at 6 month than 12 month follow up. Significant improvement in male group at 6-month post injection with statistically significant difference $(\mathrm{p}=<0.05)$. No statistically significant difference in the improvement of ADL scores between males and females during follow up period. 
Table (7): Comparison between males and females as regard WOMAC scores pre and post injection

\begin{tabular}{|c|c|c|c|c|c|c|c|c|c|}
\hline & \multicolumn{6}{|c|}{ Sex } & \multirow{2}{*}{\multicolumn{2}{|c|}{ T-Test }} \\
\hline & & \multicolumn{3}{|c|}{ Female } & \multicolumn{3}{|c|}{ Male } & & \\
\hline & & Mean & \pm & SD & Mean & \pm & SD & $\mathbf{T}$ & P-value \\
\hline \multirow{3}{*}{ 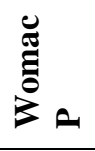 } & Before & 5.318 & \pm & 1.041 & 5.545 & \pm & 0.963 & -0.752 & 0.456 \\
\hline & After 6Months & 5.636 & \pm & 0.581 & 5.864 & \pm & 0.468 & -1.429 & 0.160 \\
\hline & After 12Months & 5.364 & \pm & 0.953 & 5.545 & \pm & 0.963 & -0.629 & 0.532 \\
\hline \multirow{3}{*}{ 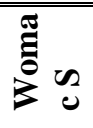 } & Before & 5.545 & \pm & 1.745 & (5.614 & \pm & 1.385 & -0.203 & 0.840 \\
\hline & After 6Months & 3.386 & \pm & 1.316 & 3.045 & \pm & 1.033 & 1.352 & 0.180 \\
\hline & After 12Months & 4.659 & \pm & 1.493 & 4.705 & \pm & 1.286 & -0.153 & 0.879 \\
\hline \multirow{3}{*}{ ن } & Before & 45.909 & \pm & 11.947 & 42.432 & \pm & 7.916 & 1.609 & 0.111 \\
\hline & After 6Months & 29.659 & \pm & 11.803 & 23.227 & \pm & 6.317 & 3.187 & $0.002 *$ \\
\hline & After 12Months & 42.023 & \pm & 12.328 & 35.068 & \pm & 6.652 & 3.293 & $0.001^{*}$ \\
\hline \multirow{3}{*}{ 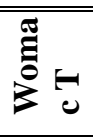 } & Before & 63.364 & \pm & 16.891 & 60.909 & \pm & 11.924 & 0.787 & 0.433 \\
\hline & After 6Months & 41.000 & \pm & 15.353 & 33.182 & \pm & 9.148 & 2.902 & $0.005^{*}$ \\
\hline & After 12Months & 58.932 & \pm & 17.266 & 50.432 & \pm & 10.429 & 2.795 & $0.006^{*}$ \\
\hline
\end{tabular}

WOMAC: Western Ontario and McMaster Universities Arthritis Index, P: pain, S: stiffness, PF: physical function.

Table (7) shows that WOMAC pain and stiffness subscales scores improved in both males and females at 6 and 12 months follow up. Males response post injection is better than female but is not of statistically significant difference $(\mathrm{P}=>0.05)$. as well as the improvement in physical function domain of WOMAC in both groups but male response is better at 6 month and 12 months follow up with high statistically significant difference $(\mathrm{P}$ $=<0.005)$

Table (8): Comparison among males and females in WOMAC subscales at baseline, after 6month and 12month post injection:

\begin{tabular}{|c|c|c|c|c|c|c|c|c|c|}
\hline \multirow{6}{*}{ 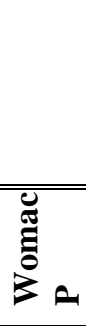 } & & \multicolumn{8}{|c|}{ Sex } \\
\hline & & \multicolumn{4}{|c|}{ Female } & \multicolumn{4}{|c|}{ Male } \\
\hline & & \multicolumn{3}{|c|}{$\begin{array}{l}\text { Differences } \\
\text { Mean } \pm \text { SD }\end{array}$} & \multirow{2}{*}{$\begin{array}{c}\text { Paired Test } \\
<0.001^{*}\end{array}$} & \multicolumn{3}{|c|}{$\begin{array}{l}\text { Differences } \\
\text { Mean } \pm \text { SD }\end{array}$} & \multirow{2}{*}{$\begin{array}{c}\text { Paired Test } \\
<0.001^{*}\end{array}$} \\
\hline & $\mathrm{B} / 6 \mathrm{M}$ & 25.045 & \pm & 1.413 & & 5.932 & \pm & 1.319 & \\
\hline & $\mathrm{B} / \mathbf{1 2 M}$ & 1.432 & \pm & 1.605 & $<0.001 *$ & 2.205 & \pm & 1.212 & $<0.001^{*}$ \\
\hline & $6 \mathrm{M} / 12 \mathrm{M}$ & -3.614 & \pm & 1.674 & $<0.001 *$ & -3.727 & \pm & 1.590 & $<0.001 *$ \\
\hline \multirow{3}{*}{ 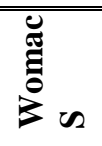 } & $\mathrm{B} / 6 \mathrm{M}$ & 2.159 & \pm & 0.776 & < <0.001* & 2.568 & \pm & 0.846 & < <0.001* \\
\hline & $\mathrm{B} / \mathbf{1 2 M}$ & 0.886 & \pm & 0.655 & $<0.001 *$ & 0.909 & \pm & 0.741 & $<0.001 *$ \\
\hline & $6 \mathrm{M} / 12 \mathrm{M}$ & -1.273 & \pm & 0.585 & $<0.001 *$ & -1.659 & \pm & 0.914 & $<0.001 *$ \\
\hline \multirow{3}{*}{ 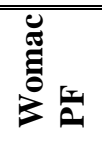 } & B/6M & 16.250 & \pm & 6.051 & $<<0.001^{*}$ & 19.205 & \pm & 5.210 & < <0.001* \\
\hline & $\mathrm{B} / \mathbf{1 2 M}$ & 3.886 & \pm & 2.982 & $<0.001 *$ & 7.364 & \pm & 3.730 & $<0.001 *$ \\
\hline & 6M/12M & -12.364 & \pm & 5.063 & $<0.001 *$ & -11.841 & \pm & 3.735 & $<0.001 *$ \\
\hline \multirow{3}{*}{ 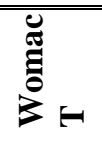 } & $\mathrm{B} / 6 \mathrm{M}$ & 22.364 & \pm & 7.898 & $<<0.001 *$ & 27.727 & \pm & 6.013 & $<<0.001 *$ \\
\hline & $\mathrm{B} / \mathbf{1 2 M}$ & 4.432 & \pm & 6.919 & $<0.001 *$ & 10.477 & \pm & 4.516 & $<0.001 *$ \\
\hline & $6 \mathrm{M} / 12 \mathrm{M}$ & -17.932 & \pm & 6.450 & $<0.001 *$ & -17.250 & \pm & 4.847 & $<0.001 *$ \\
\hline
\end{tabular}

*WOMAC: Western Ontario and McMaster Universities Arthritis Index, P: pain, S: stiffness, PF: physical function, B: before, M: months.

Table (8) compares baseline, 6 months and 12 months scores of all WOMAC subscales scores among males and females groups and shows that a highly significant improvement ( $\mathrm{P}=<0.001)$ occurred in all WOMAC subscales scores (pain, stiffness and physical function) among subjects in both groups at 6 month and 12 month follow up post injection. this significant improvement is better at 6 months follow up than 12 months follow up point with high statistically significant difference $(\mathrm{P}=<0.001)$. 
Table (9): OA grades among study group as regard age, height, weight and BMI

\begin{tabular}{|c|c|c|c|c|c|c|c|c|c|c|c|c|}
\hline & \multicolumn{9}{|c|}{ OA grade } & \multirow{2}{*}{\multicolumn{2}{|c|}{ ANOVA }} \\
\hline & & \multicolumn{3}{|c|}{ Grade I } & \multicolumn{3}{|c|}{ Grade II } & \multicolumn{3}{|c|}{ Grade III } & & \\
\hline & & $\begin{array}{c}\text { Mea } \\
\mathbf{n}\end{array}$ & \pm & SD & Mean & \pm & SD & Mean & \pm & SD & $\mathbf{F}$ & $\begin{array}{c}\text { P- } \\
\text { value }\end{array}$ \\
\hline \multicolumn{2}{|l|}{ Age } & 64.12 & \pm & 3.834 & 63.35 & \pm & 4.242 & 64.73 & \pm & 4.121 & 0.506 & 0.607 \\
\hline \multicolumn{2}{|l|}{ Height } & 166.6 & \pm & 6.413 & 166.94 & \pm & 6.139 & 169.15 & \pm & 7.522 & 0.629 & 0.538 \\
\hline \multirow{3}{*}{ Weight } & Before & 81.87 & \pm & 8.839 & 81.47 & \pm & 6.793 & 83.68 & \pm & 8.433 & 0.382 & 0.685 \\
\hline & After 6 Months & 81.87 & \pm & 8.839 & 81.05 & \pm & 6.878 & 83.65 & \pm & 8.209 & 0.510 & 0.604 \\
\hline & After 12 Months & 81.75 & \pm & 8.876 & 80.70 & \pm & 6.669 & 83.52 & \pm & 8.208 & 0.602 & 0.553 \\
\hline \multirow{3}{*}{ BMI } & Before & 29.78 & \pm & 1.533 & 29.21 & \pm & 2.711 & 29.26 & \pm & 2.991 & 0.136 & 0.873 \\
\hline & After 6 Months & 29.78 & \pm & 1.533 & 29.08 & \pm & 2.776 & 29.25 & \pm & 2.932 & 0.190 & 0.828 \\
\hline & After 12 Months & 29.73 & \pm & 1.554 & 28.94 & \pm & 2.745 & 29.24 & \pm & 2.959 & 0.237 & 0.790 \\
\hline
\end{tabular}

*OA: osteoarthritis, BMI: body mass index.

Table (9) shows that there is no significant difference in age, height, weight, and BMI

between different groups of OA grade, as well as after 6 and 12 months follow up.

Table (10): OA grades and ADL scores at baseline, 6 months and 12 months follow up

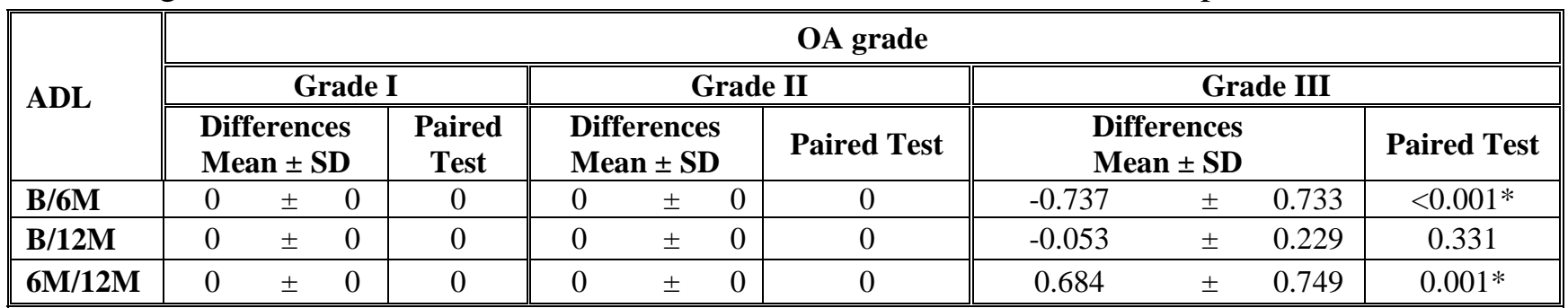

*OA: osteoarthritis, ADL: activities of daily living, B: before, M: months.

Table (10) shows that when comparing ADL scores between baseline and at both points follow up among different OA grades:

No difference in ADL scores occurred among subjects with grade 1 and 2 OA during follow period. Significant improvement in ADL scores among subjects with grade 3 OA achieved at 6 months follow up post PRP injection $(\mathrm{P}=<$ $0.001)$.

Table (11): OA grade and TUG scores at baseline, 6 months and 12 months follow up

\begin{tabular}{|c|c|c|c|c|c|c|c|c|c|c|c|}
\hline \multirow[b]{3}{*}{ TUG } & \multicolumn{6}{|c|}{ OA grade } & \multicolumn{2}{|c|}{ ANOVA } & \multicolumn{3}{|c|}{ TUKEY'S Test } \\
\hline & \multicolumn{2}{|c|}{ Grade I } & \multicolumn{2}{|c|}{ Grade II } & \multicolumn{2}{|c|}{ Grade III } & \multirow[b]{2}{*}{$\mathbf{F}$} & \multirow[b]{2}{*}{$\begin{array}{c}P- \\
\text { value }\end{array}$} & \multirow[b]{2}{*}{ I\&II } & \multirow[b]{2}{*}{ I\&III } & \multirow[b]{2}{*}{$\begin{array}{c}\text { II\&II } \\
\text { I }\end{array}$} \\
\hline & $\begin{array}{c}\text { Differenc } \\
\text { es } \\
\text { Mean } \pm \\
\text { SD }\end{array}$ & $\begin{array}{c}\text { Paire } \\
\text { d } \\
\text { Test }\end{array}$ & $\begin{array}{c}\text { Differenc } \\
\text { es } \\
\text { Mean } \pm \\
\text { SD }\end{array}$ & $\begin{array}{c}\text { Paired } \\
\text { Test }\end{array}$ & $\begin{array}{c}\text { Differenc } \\
\text { es } \\
\text { Mean } \pm \\
\text { SD }\end{array}$ & $\begin{array}{c}\text { Paired } \\
\text { Test }\end{array}$ & & & & & \\
\hline $\mathrm{B} / 6 \mathrm{M}$ & $\begin{array}{c}2.250 \pm \\
1.282\end{array}$ & $\begin{array}{c}0.002 \\
*\end{array}$ & $\begin{array}{c}4.235 \\
\pm 1.147\end{array}$ & $\begin{array}{c}<0.001 \\
*\end{array}$ & $\begin{array}{c}4.684 \pm \\
1.493\end{array}$ & $\begin{array}{c}<0.001 \\
*\end{array}$ & $\begin{array}{c}9.62 \\
9\end{array}$ & $\begin{array}{c}<0.001 \\
*\end{array}$ & $\begin{array}{c}0.003 \\
*\end{array}$ & $\begin{array}{c}<0.001 \\
*\end{array}$ & 0.575 \\
\hline $\mathrm{B} / \mathbf{1 2 M}$ & $\begin{array}{c}1.250 \pm \\
1.282\end{array}$ & $\begin{array}{c}0.028 \\
*\end{array}$ & $\begin{array}{c}2.059 \\
\pm 1.249\end{array}$ & $\begin{array}{c}<0.001 \\
*\end{array}$ & $\begin{array}{c}2.105 \pm \\
1.663\end{array}$ & $\begin{array}{c}<0.001 \\
*\end{array}$ & $\begin{array}{c}1.08 \\
5\end{array}$ & 0.347 & & & \\
\hline $6 M / 12 M$ & $\begin{array}{c}-1.00 \pm \\
0.926\end{array}$ & $\begin{array}{c}0.018 \\
* \\
\end{array}$ & $\begin{array}{c}-2.176 \pm \\
1.185 \\
\end{array}$ & $\begin{array}{c}<0.001 \\
*\end{array}$ & $\begin{array}{c}-2.579 \pm \\
1.121\end{array}$ & $0.001 *$ & $\begin{array}{c}5.64 \\
8\end{array}$ & $0.007 *$ & $\begin{array}{c}0.047 \\
*\end{array}$ & $0.005^{*}$ & 0.532 \\
\hline
\end{tabular}

*OA: osteoarthritis, TUG: timed up and go test, B: before, M: months.

Table (11) shows that when comparing differences in TUG scores in response to PRP injection at baseline and both points follow up among different OA grades:
Significant improvement achieved at both 6 and 12 months follow up, this improvement was better among subjects with grade 3 then grade 2 OA than 
grade $1 \mathrm{OA}$ with high statistically significant difference at 6 months follow up.

However, at 12 months follow up the improvement show no significant difference between different OA grades. This reflects the deterioration of response among grade 3 then grade 2 OA subjects after 12-month post injection.

Table (12): OA grade and NRS scores at baseline, 6 months and 12 months follow up

\begin{tabular}{|c|c|c|c|c|c|c|c|c|c|c|c|c|c|c|}
\hline \multirow{4}{*}{$\begin{array}{l}\text { NRS } \\
\text { B/6M }\end{array}$} & \multicolumn{12}{|c|}{ OA grade } & \multicolumn{2}{|c|}{ ANOVA } \\
\hline & \multicolumn{4}{|c|}{ Grade I } & \multicolumn{4}{|c|}{ Grade II } & \multicolumn{4}{|c|}{ Grade III } & \multirow[b]{2}{*}{$\mathbf{F}$} & \multirow[b]{2}{*}{$\begin{array}{c}P \text { - } \\
\text { value }\end{array}$} \\
\hline & \multicolumn{3}{|c|}{$\begin{array}{l}\text { Differences } \\
\text { Mean } \pm \text { SD }\end{array}$} & \multirow{2}{*}{$\begin{array}{c}\text { Paired Test } \\
<0.001^{*}\end{array}$} & \multicolumn{3}{|c|}{$\begin{array}{l}\text { Differences } \\
\text { Mean } \pm \text { SD }\end{array}$} & \multirow{2}{*}{$\begin{array}{c}\begin{array}{c}\text { Paired } \\
\text { Test }\end{array} \\
<0.001^{*}\end{array}$} & \multicolumn{3}{|c|}{$\begin{array}{l}\text { Differences } \\
\text { Mean } \pm \text { SD }\end{array}$} & \multirow{2}{*}{$\begin{array}{c}\begin{array}{c}\text { Paired } \\
\text { Test }\end{array} \\
<0.001 * \\
\end{array}$} & & \\
\hline & 4.25 & \pm & 0.707 & & 4.82 & \pm & 0.809 & & 5.00 & \pm & 0.943 & & 2.181 & 0.126 \\
\hline $\mathrm{B} / \mathbf{1 2 M}$ & 0.87 & \pm & 0.641 & $0.006^{*}$ & 1.17 & \pm & 0.728 & $<0.001 *$ & 1.15 & \pm & 0.958 & $<0.001 *$ & 0.412 & 0.665 \\
\hline $6 \mathrm{M} / 12 \mathrm{M}$ & -3.37 & \pm & 0.744 & $<0.001 *$ & $3 . \overline{-}$ & \pm & 0.931 & $<0.001 *$ & $\begin{array}{c}- \\
3.84\end{array}$ & $I$ & 0.898 & $0.001 *$ & 0.801 & 0.456 \\
\hline
\end{tabular}

*OA: osteoarthritis, NRS: numeric rating scale, B: before, M: months.

Table (12) shows that when comparing the differences in response of NRS scores at baseline and both points follow up:

Significant improvement in pain scores among all grades of knee OA, the best improvement achieved at 6 months follow up.Rate of improvement in pain Scores at 6 and 12 months follow up was almost equal between the 3 grades of OA with no statistical significant difference.

Table (13): Linear multi-regression analysis WOMAC scores at 6 months follow up:

\begin{tabular}{|l|c|c||}
\hline $\begin{array}{c}\text { WOMAC } \\
\text { (6 months) }\end{array}$ & T & P-value \\
\hline Age & 1.760 & 0.082 \\
\hline Height 6m & 0.606 & 0.546 \\
\hline Weight 6m & -0.925 & 0.358 \\
\hline BMI 6m & 0.445 & 0.658 \\
\hline Sex & -4.933 & $<0.001^{*}$ \\
\hline OA grade & 9.564 & $<0.001^{*}$ \\
\hline
\end{tabular}

*WOMAC: Western Ontario and McMaster Universities Arthritis Index, BMI: body mass index, OA: osteoarthritis

Table (13) shows that OA grade and sex independently significantly affect WOMAC scores in response to PRP injection with high statistically significant difference $(\mathrm{p}=<0.001)$.

Table (14): Linear multi-regression analysis WOMAC scores at 12 months follow up

\begin{tabular}{|l|c|c||}
\hline $\begin{array}{l}\text { WOMAC } \\
\text { (12 months) }\end{array}$ & T & P-value \\
\hline Age & .218 & 0.828 \\
\hline Height 6m & -1.468 & 0.146 \\
\hline Weight 6m & .144 & 0.886 \\
\hline BMI 6m & -1.004 & 0.318 \\
\hline Sex & -2.609 & $0.011^{*}$ \\
\hline OA grade & 4.643 & $<0.001^{*}$ \\
\hline
\end{tabular}

*WOMAC: Western Ontario and McMaster Universities Arthritis Index, BMI: body mass index, OA: osteoarthritis

Table (14) shows that OA grade and sex independently significantly affect WOMAC scores in response to PRP injection with high statistically significant difference $(p=<0.005)$.

Table (15): linear multi-regression analysis TUG scores at 6 and 12 months follow up:

Table (15a):

\begin{tabular}{|l|c|c||}
\hline $\begin{array}{l}\text { TUG } \\
\text { (6 months) }\end{array}$ & T & P-value \\
\hline Age & .872 & 0.388 \\
\hline Height 6m & 1.569 & 0.125 \\
\hline Weight 6m & -1.562 & 0.127 \\
\hline BMI 6m & 1.414 & 0.165 \\
\hline Sex & -2.496 & $0.017^{*}$ \\
\hline OA grade & 6.349 & $<0.001^{*}$ \\
\hline
\end{tabular}

*TUG: timed up and go, BMI: body mass index, OA: osteoarthritis 


\begin{tabular}{|l|c|c|}
\hline $\begin{array}{l}\text { TUG } \\
\text { (12 months) }\end{array}$ & T & P-value \\
\hline Age & .731 & 0.469 \\
\hline Height 12ms & 1.193 & 0.240 \\
\hline Weight 12ms & -1.192 & 0.241 \\
\hline BMI 12ms & 1.044 & 0.303 \\
\hline Sex & -3.226 & $0.003^{*}$ \\
\hline OA grade & 7.017 & $<0.001^{*}$ \\
\hline
\end{tabular}

*TUG: timed up and go, BMI: body mass index, OA: osteoarthritis

Table (15 a \& b) shows that OA grade and sex each independently significantly affect TUG scores at the 6th and 12th months post PRP injection with high statistically significant difference $(\mathrm{p}=<0.005)$.

Table (16): linear multi-regression analysis NRS scores at 6 and 12 months follow up:

Table (16 a):

\begin{tabular}{|l|c|c|}
\hline $\begin{array}{l}\text { NRS } \\
\text { (6 months) }\end{array}$ & T & P-value \\
\hline Age & .497 & 0.622 \\
\hline Height 6ms & -.956 & 0.345 \\
\hline Weight 6ms & .786 & 0.437 \\
\hline BMI 6ms & -1.188 & 0.242 \\
\hline Sex & -4.173 & $<0.001^{*}$ \\
\hline OA grade & 5.951 & $<0.001^{*}$ \\
\hline
\end{tabular}

*NRS: numeric rating scale, BMI: body mass index, OA: osteoarthritis

Table (16b):

\begin{tabular}{|l|c|c||}
\hline $\begin{array}{l}\text { NRS } \\
\text { (12 months) }\end{array}$ & T & P-value \\
\hline Age & .797 & 0.430 \\
\hline Height 12 ms & -1.900 & 0.065 \\
\hline Weight 12ms & 1.712 & 0.095 \\
\hline BMI 12ms & -2.145 & $0.038^{*}$ \\
\hline Sex & -3.503 & $0.001^{*}$ \\
\hline OA grade & 4.992 & $<0.001^{*}$ \\
\hline
\end{tabular}

*NRS: numeric rating scale, BMI: body mass index, OA: osteoarthritis

Table (16 a \& b) shows that OA grade and sex each independently significantly affect NRS scores at the 6th and 12th months post PRP injection with high statistically significant difference $(\mathrm{p}=<0.005)$. BMI independently significantly affect NRS scores at the 12th month.

\section{Discussion}

Knee osteoarthritis is a chief health issue correlated to aging, pathologically described by articular cartilage progressive loss causing pain, functional impairment, and disability with considerable restricting impact on patient's daily activities consequently diminishing patient's quality of life. $^{15}$

Platelet-rich plasma (PRP) is one of various developments within regenerative field of medicine. It is a natural concentrate of autologous growth factors from the blood, allows in a simple, low cost and minimally invasive way to obtain a concentration of many growth factors which act by repairing as well as replacing pathologic, damaged, and aged tissue. ${ }^{16}$

The current research study recruited forty-four subjects divided into two equal research groups of 22 male and 22 female patients both groups showed no significant differences as regard age, weight, and OA grade. All had positive X-ray findings suggestive of mild to moderate knee OA according to Kellgren-Lawrence Grading Scale. Subject's age ranged from 60 to 70 years old with mean age 64 . They were recruited randomly from outpatient clinics and inpatients wards of Ain Shams University Hospital. Their knees were injected by 2-3 $\mathrm{ml}$ of PRP in a single session and followed up clinically for 12 months.

Functional assessments of the patients were done at baseline evaluation, 6 and 12 months after PRP injection using the Western Ontario and McMaster Universities Arthritis Index (WOMAC), Timed up and go (TUG) and activity of daily livings (ADL). Whereas, Quantitative assessment of pain intensity was done using numeric pain rating scale (NRS-11).

No major adverse events related to the injections were observed during the treatment and follow-up period. In only one case, a patient experienced marked pain with swelling after the injection, which spontaneously resolved after 2 weeks. In nearly 15 subjects' slight pain during first 2 or 3 days was reported. 
On studying the effect of PRP injection on functional assessment in elderly patients with knee OA using WOMAC tool and its subscales (pain, stiffness and physical function) scores, standing and walking abilities using activities of daily living (ADL) and timed up and go tests (TUG) and subjective pain measurement using the Numerical Rating Scale (NRS). The research team of investigators observed significant improvement in all assessment scores after 6 months of follow up. Furthermore, the current research study results revealed and displayed that improvement accomplished after 12 months of follow up was statistically significant in comparison to baseline assessment scores; on the other hand this improvement was much more less than scores achieved after 6 months of injection. Those research findings could be justified that there is gradual loss in the clinical efficacy levels of PRP injections occurred over the period of follow up which lasted for one year (12 months), on the other hand this improvement remains statistically significant when compared to baseline scores.

A prior research group of investigators mentioned that estimated clinical efficacy and benefits of PRP injections were limited by time, and the estimated duration of effectiveness was at 1 year in harmony with the current research study in which they recruited a total of 33 cases that received 2 dosages of PRP injections. Significant improvement in all functional and pain assessment scores after 6 and 12 months follow up with tendency for gradual reduction in clinical efficacy after 12 months of follow up. ${ }^{17}$

Another research team priorly conducted a study like the current research in approach and methodology in which a total of 102 research study subjects were administered different dosages of PRP injections, their WOMAC and TUG scores were significantly better at 6 months than pre injection scores. ${ }^{18}$

Another previous research study conducted on ninety-six study subjects with mean age of 63.6 years, revealed and displayed significant improvement in WOMAC scores in comparison to baseline scores. Even though this improvement remained statistically significant until study completion but reduction in the clinical efficacy after 12 months had occurred. ${ }^{19}$

On contrary a research study previously conducted that enrolled 100 cases having a mean age of 47 years and treated with 3 sessions of PRP injections. using IKDC functional assessment, significant clinical improvement achieved after 6 months of follow up, then functional scores became significantly worse at the 12th month of follow-up $(\mathrm{P}$ value $=0.02){ }^{20}$

Another research group of investigators revealed and displayed among their research findings that the long term impact of PRP injections in knee OA patients in which all of the evaluated functional parameters among study subjects have shown a marked worsening over time, with a statistically significantly lower score at the at end point of follow-up. ${ }^{21}$

The controversy in results of the clinical efficacy and duration of PRP injections could be justified by the fact that there is variations in PRP products preparation techniques and centrifugation, platelets concentrations, presence of leukocytes and erythrocytes and difference in number of injections and their intervals as well as patient selection criteria that could have caused different results in various clinical applications. ${ }^{22,23}$

The current research study findings our research team did not observe any statistically significant correlation between age, weight, BMI and the clinical effect of PRP. Multilinear regression analysis revealed that the degree of severity of knee OA and gender difference were the most important factors affecting the response to PRP intraarticular treatment.

Furthermore, in the current research by evaluation of the impact of gender differences on response to PRP intraarticular injections on functional and pain outcomes in knee OA patients. Our study included 22 males and 22 females who were matched in age, weight, and OA grade but females were higher in their BMI (mean = 30.8) than males $($ mean $=27.8)$. Even though female patients 
in current research study had higher BMI than male patients, but no statistically significant differences were revealed at baseline scores either for functional assessment (WOMAC, TUG and ADL) or pain (NRS) assessment tool scores.

Male patients showed better results in most of the assessment tools than females in response to PRP injections at 6 and 12 months of follow up, however this improvement was not of any statistically significant difference in functional assessment as regard ADL, WOMAC (pain and stiffness) scores. A statistically significant improvement among males in comparison to females was noticed in WOMAC (physical function and total) scores after 6- and 12-months post PRP injections, TUG scores at 12 months and NRS pain score at 6 months.

Our results could be justified by the fact that many factors could coexist and result in the poor responsiveness to PRP injections among patients such as lack of physical activity, diminished mobility, muscle weakness and high body mass index which is more common among females.

Similarly, another research team have reported that the unfavorable clinical results after single session of intraarticular PRP injection, lesser improvement among female patients. Those findings could be justified by gender-specific biological and biomechanical features, that affect the etiopathogenesis, the effects of the growth factors and ultimately, the clinical response to treatment. $^{24}$

Furthermore, another research team of investigators have observed interestingly that their patients for 12 months after PRP treatment and showed worse clinical outcomes were seen among women and in patients with higher BMI.

Additionally, it was observed in a prior research study similar to the current research that there was no correlation of all WOMAC parameters and VAS mean scoring level with sex, on the other hand male patients had an earlier response in comparison to female patients. ${ }^{25}$

On contrary it was observed by other research groups in previous studies that there was no statistically significant impact of sex on WOMAC and IKDC functional assessment scores or VAS score for pain assessment after 6 months of treatment. Additionally, it was shown that Clinical improvement in WOMAC and NRS did not correlate with gender throughout the 24 months of follow up. ${ }^{26}$

In the studies conducted so far, the lack of standardization of PRP dosing regimens, follow up duration, different assessment tools and sampling of studied population makes it difficult to compare outcomes of studies for the evaluation of clinical effectiveness of intraarticular PRP injection.PRP treatment results differ from patient to patient according to individual variability, including baseline platelet count, level of chondropenia, patient's activity level, underlying medical history, inflammatory state, immune response, age, hormone and nutritional status and psychosocial factors. ${ }^{2,5,10}$

A restriction in the current study is the lack of control group, which is an obstacle in reaching definitive conclusions. In addition to relatively the small sample size included. Also, we followed our patients for a minimum of only 12 months; longterm follow-up may provide more valuable results. Another limitation was the lack of imaging assessment to evaluate OA progression either MRI or ultrasonography. MRI was not performed because of high costs but could have provided more objective data about the benefit of treatment. $^{13,16,20}$

\section{Conclusion and Recommendations}

Intra-articular injection of PRP in knee OA may be a valid alternative treatment for knee osteoarthritis. Intra-articular infiltration of autologous PRP under the conditions described in this study seems to be a safe, effective treatment for knee OA and does not require hospitalization or surgery.PRP is an effective alternative treatment options for knee OA in both males and females taking in consideration the important coexisting factor that affect the response to PRP Intra-articular injection of PRP into the 
osteoarthritic knee improves function and pain of the knee joint with no associated systemic repercussions or complications; however controversy regarding the optimum PRP amount and frequency of injections is still problematic.

These results are promising to encourage large randomized clinical trials, and we are optimistic about this new step for the treatment of knee osteoarthritis.

PRP intraarticular therapy should be considered as an alternative therapy in management of knee OA among the elderly. Future clinical trials should be concerned with differences in PRP products preparation techniques and centrifugation, platelets concentrations, presence of leukocytes and erythrocytes as well as demonstrating if a single injection is sufficient or multiple injections might be required and intervals between injections for satisfying results. Further clinical trials including larger number of studied subjects with respect to equal distribution of different $\mathrm{OA}$ grades. Future clinical trial focusing on gender difference effect on response to PRP injections with careful selection of subjects and documentation of different factors influencing the clinical response. The post injection followup should be lasting for a long duration to demonstrate the long-term clinical efficacy and adverse effects of PRP in knee OA. More objective parameters to be used like joint inflammatory biochemical markers or biomechanical studies to determine the clinical improvement rather than the subjective assessment tools. Radiographic imaging assessment should be included to evaluate OA progression and provide objective data on clinical improvement of cartilage quality.

Disclosure of interest: The authors declare that there is no conflict of interest associated with this manuscript.

Funding: This study received no financial support.

Acknowledgements: The authors highly appreciate the support from Ain Shams University
Hospital for the outstanding support during the entire experiment. The manuscript is based on thesis held in Ain Shams University Hospital. http://srv4.eulc.edu.eg/eulc_v5/Libraries/Thesis/B rowseThesisPages.aspx? $\mathrm{fn}=$ PublicDrawThesis\&B ibID $=12436193$

\section{References}

1. Anitua E, Prado R, Padilla S, et al. (2016). Platelet-rich plasma therapy: another appealing technology for regenerative medicine? Regen Med. Jun; 11(4):355-7.

2. Duymus TM, Mutlu S, Dernek B, et al., (2017). Choice of intra-articular injection in treatment of knee osteoarthritis: plateletrich plasma, hyaluronic acid or ozone options. Knee Surg Sports Traumatol Arthrosc. Feb; 25(2):485-492.

3. Castell M V, Pas S, Otero A, et al (2015). Osteoarthritis and frailty in elderly individuals across six European countries: results from the European Project on Osteoarthritis (EPOSA); BMC Musculoskelet Disord. 16: 351-359.

4. Chang KV, Hung CY, Aliwarga F, et al., (2014). Comparative effectiveness of platelet-rich plasma injections for treating knee joint cartilage degenerative pathology: a systematic review and metaanalysis. Arch Phys Med Rehabil. Mar; 95(3):562-75.

5. Cole B.J., Karas V, Hussey K., et al., (2016). Hyaluronic Acid Versus PlateletRich Plasma: A Prospective, Double-Blind Randomized Controlled Trial Comparing Clinical Outcomes and Effects on IntraArticular Biology for the Treatment of Knee Osteoarthritis Am J Sports Med Oct 21: 45 (2), 339-346.

6. Forogh B, Mianehsaz E, Shoaee S, et al. (2016). Effect of single injection of platelet-rich plasma in comparison with corticosteroid on knee osteoarthritis: a double-blind randomized clinical trial. J Sports Med Phys Fitness.; 56(7-8):901-8. 
7. Freitag J, Bates D, Boyd R, et al (2016). Mesenchymal stem cell therapy in the treatment of osteoarthritis: reparative pathways, safety and efficacy - a review. BMC Musculoskeletal Disorders. 17, 230. doi:10.1186/s12891-016-1085-9

8. Boswell SG, Schnabel LV, Mohammed $\mathrm{HO}$, et al. (2014). Increasing platelet concentrations in leukocyte-reduced platelet-rich plasma decrease collagen gene synthesis in tendons. Am J Sports Med. 42(1):42-9.

9. Boyan, B. D., Tosi, L. L., Coutts, R. D., et al. (2013). Addressing the gaps: sex differences in osteoarthritis of the knee. Biology of Sex Differences, 4, 4.

10. Braun HJ, Kim HJ, Chu CR, et al. (2014). The effect of platelet-rich plasma formulations and blood products on human synoviocytes: implications for intraarticular injury and therapy. Am J Sports Med. 42(5):1204-10

11. Davis VL, Abukabda AB, Radio NM, et al. (2014). Platelet-rich preparations to improve healing. Part I: workable options for every size practice. J Oral Implantol; 40(4):500-10.

12. Cooper, C., Rannou, F., Richette, P., et al. (2017). Use of Intra-Articular Hyaluronic Acid in the Management of Knee Osteoarthritis in Clinical Practice. Arthritis Care \& Research. Accepted Author Manuscript. doi:10.1002/acr.23204

13. Dold AP, Zywiel MG, Taylor DW et al, (2014).Theodoropoulos J. Platelet-rich plasma in the management of articular cartilage pathology: a systematic review. Clin J Sport Med. 24:31-43

14. Musumeci G, Szychlinska MA and Mobasheri A (2015). Age related degeneration of articular cartilage in the pathogenesis of osteoarthritis: molecular markers of senescent chondrocytes. Histol Histopathol 30: 1-12.
15. Çalış HT, Sutbeyaz ST, Guler E, et al. (2015). Efficacy of intra-articular autologous platelet rich plasma application in knee osteoarthritis. Arch Rheumatol. 30: 198-205

16. Castañeda S, Roman-Blas JA, Largo R, et al, (2014). Osteoarthritis: a progressive disease with changing phenotypes. Rheumatology (Oxford). Jan; 53(1):1-3.

17. Guo Y, Yu H, Yuan L, et al; (2016). Treatment of knee osteoarthritis with platelet-rich plasma plus hyaluronic acid in comparison with platelet-rich plasma only. Int J Clin Exp Med; 9(6):12085-12090

18. Hassan AS, El-Shafey AM,. Ahmed HS, et al., (2015). Effectiveness of the intraarticular injection of platelet rich plasma in the treatment of patients with primary knee osteoarthritis. The Egyptian Rheumatologist 37(3): 119-124.

19. Lana J F S D, Vicente E F, Weglein A, et al, (2016). Platelet Rich Plasma in Musculoskeletal Practice, Platelet-Rich Plasma in Pain Medicine. Springer-Verlag, London. Chapter 2; pp 30- 62.

20. Filardo G, Di Matteo B, Di Martino A, et al., (2015). Platelet-Rich Plasma IntraArticular Knee Injections Show No Superiority Versus Viscosupplementation: A Randomized Controlled Trial. Am J Sports Med.; 43(7):1575-82

21. Filardo G, Kon E, Roffi A, et al, (2015). Platelet-rich plasma: why intra-articular? A systematic review of preclinical studies and clinical evidence on PRPfor joint degeneration. Knee Surg Sports Traumatol Arthrosc. 23(9):2459-2474.

22. Hunter D (2014). Atlas of Osteoarthritis; Epidemiology of osteoarthritis, Springer Healthcare, London, UK. Chapter 6; pp 83:99.

23. Iaccarino MA and Borg-Stein J (2016). Platelet Rich Plasma in Musculoskeletal Practice. Platelet-Rich Plasma in Knee 
Osteoarthritis in the Athlete. Chapter 6, pp 123-146.

24. Joshi Jubert N., Rodríguez, L., RevertéVinaixa, M. M., et al. (2017). Platelet-Rich Plasma Injections for Advanced Knee Osteoarthritis: A Prospective, Randomized, Double-Blinded Clinical Trial. Orthopaedic Journal of Sports Medicine, 5(2), 2325967116689386.

25. Kavadar G, Demircioglu DT., Celik MY., ET AL., (2015). Effectiveness of plateletrich plasma in the treatment of moderate knee osteoarthritis: a randomized prospective study. J Phys Ther Sci. Dec; 27(12): 3863-3867.

26. Kawano M M, Araújo I L, Castro M C, et al. (2015). Assessment of quality of life in patients with knee osteoarthritis. Acta OrtopedicaBrasileira, 23(6), 307-310. 